\title{
API: GERAÇÃO AUTOMÁTICA DE DESENHOS DE PORMENOR DE ARMADURAS EM ESTRUTURAS DE BETÃO
}

\author{
Ruben Pereira $^{(1)}$, Rodrigo Falcão Moreira ${ }^{(1)(2)}$ \\ (1) GROUNDMOTION - Earthquake \& Structural Engineering International, Lda, Porto \\ (2) CONSTRUCT-LESE, Faculty of Engineering, University of Porto
}

\begin{abstract}
Resumo
O avanço significativo da construção digital, concretizado na metodologia BIM (Building Information Modelling), veio abrir portas a projetos e empreendimentos cada vez mais complexos. Essa complexidade é já uma realidade e tem influência significativa nas tarefas de análise estrutural e definição de processos construtivos. Em complemento, existe a necessidade premente de automatizar aquelas que são as tarefas mais repetitivas no desenvolvimento de um projeto. Essa automatização, desde que devidamente validada, permite a redução de erros e o controlo rigoroso e imediato de todas as tarefas.

Neste enquadramento, o presente artigo aborda a temática na perspetiva do projeto de estruturas de betão, em que a tarefa de representação de armaduras é morosa e pode revelar-se demasiado "time consuming". A utilização de modelos BIM na sua pormenorização detalhada permite a verificação de incompatibilidades na modelação, criação de desenhos de pormenor, extração de quantidades e elaboração de mapas de ferros (shop drawings).

A abordagem aqui apresentada baseia-se no desenvolvimento e aplicação de uma ferramenta BIM focada na importação e tratamento da informação integrante do modelo digital. Esta ferramenta, desenvolvida em linguagem de programação C\#, funciona como um Add-in ao software primário Autodesk Revit $囚$. Incorpora uma interface gráfica e atua fundamentalmente no domínio da geração automática de desenhos de armaduras. $\mathrm{O}$ algoritmo desenvolvido vem colmatar algumas das lacunas de automatização do software primário, permitindo assim implementar um procedimento para pormenorização de armaduras de acordo com templates empresariais padronizados.
\end{abstract}

\section{Introdução}

A indústria de Arquitetura, Engenharia e Construção (AEC) há muito tempo que procura técnicas tanto para reduzir o custo e tempo de entrega, como para aumentar a produtividade e a qualidade dos projetos. A metodologia BIM tem o potencial para atingir esses objetivos [1]. 
A utilização de ferramentas que operam em ambiente BIM para a modelação de armaduras em elementos de betão armado permite uma melhor quantificação de um dos aspetos mais importantes num projeto de estruturas [2]. No entanto, a tarefa de modelar consome muito tempo e exige esforço assinalável, por vezes com o único objetivo de satisfazer um determinado nível de detalhe (LOD).

A modelação e pormenorização de armaduras deve ser ponderada para cada projeto mediante a exigência do cliente. Caso se pretenda uma rigorosa orçamentação das armaduras e a produção de shop drawings (mapas de ferros), por exemplo para projetos internacionais, a utilização de ferramentas BIM agiliza esse processo. Existem atualmente projetos que apenas recorrem à informação do modelo digital sem a necessidade de desenhos de pormenor. Em projetos em que o prazo é consideravelmente curto, a modelação completa de armaduras dificulta o cumprimento desse objetivo.

No contexto nacional existe ainda a necessidade de fornecer desenhos de pormenor de armaduras, sendo muitas vezes suficiente apresentar cortes tipo das secções dos elementos consoante a fase do projeto. A inclusão de alçados e cortes de armaduras para cada elemento em fase de execução justifica-se caso a jusante essa informação seja utilizada para fabricação/ construção de forma a economizar tempo e recursos.

O presente artigo tem como objetivo apresentar um aplicativo informático que agiliza a geração de armaduras assim como a pormenorização das mesmas. $\mathrm{O}$ aplicativo cria armaduras com base em inputs mínimos de forma a tornar rápida a sua utilização, produz automaticamente desenhos de pormenor e apresenta-os de acordo com os templates padronizados da empresa que o desenvolveu (GROUNDMOTION - Earthquake \& Structural Engineering International). Esta metodologia possibilita a rápida criação de shop drawings e bar bending schedules (tabelas onde são especificados os comprimentos de dobragem das armaduras) de acordo com a norma inglesa BS 8666:2005 [3].

A grande maioria dos atuais softwares BIM dispõem de API's que permitem a programação de algoritmos. Estes podem funcionar como add-ins tendo como propósito a implementação e automatização de tarefas de modelação, potenciando a adição das mais diversas competências ao software base. O software utilizado foi o Autodesk Revit®, que na sua API suporta a framework .NET 4.7 [4], e a linguagem de programação escolhida foi o C\#.

$\mathrm{O}$ aplicativo funciona a partir da consideração de um modelo BIM, realizado no software Autodesk Revit@, cujos objetos / famílias se encontram organizados por categorias. No caso presente, os elementos adotados foram pilares em betão armado, sendo que se pretende que o aplicativo funcione futuramente com outros elementos.

\section{Objetivos}

O nível de maturação e implementação que a metodologia BIM apresenta no contexto nacional da indústria AEC obriga ainda à conversão de toda a informação digital em documentação 2D materializada em formatos passíveis de serem compreendidos, analisados e verificados por todos os intervenientes do processo [5]. Ora, estes formatos são aqueles que ainda hoje fazem parte do processo tradicional, nomeadamente $. x l s, . p d f, . d o c, . d w g$ ou $d w f$.

Consequentemente, e tendo em conta que a nível interno o procedimento implementado na GROUNDMOTION assenta fundamentalmente na metodologia BIM, surgiu a necessidade de automatizar algumas destas tarefas de "conversão de informação" evitando qualquer perda de 
legibilidade e representatividade da informação. Nesta perspetiva sinalizaram-se aquelas que, no âmbito do projeto de estruturas, seriam as mais sensíveis e repetitivas para que de uma forma estruturada se conseguisse conceber métodos e estratégias conducentes com uma eficaz modelação e gestão dessa informação. Entre as tarefas mais sensíveis destacam-se, claramente, a geração automática de peças desenhadas.

A modelação de armaduras pode ser efetuada recorrendo às ferramentas nativas do software ou agilizada através de add-ins. Qualquer das opções auxilia na geração de armaduras. No entanto, na pormenorização das armaduras (alçados ou cortes) será necessário complementar manualmente a informação com diferentes anotações (dimensões, textos ou tags). Estas limitações obrigam a um conjunto de passos incompatível com a automatização pretendida para o efeito. Portanto, sobra o recurso ao API do software como única alternativa viável para alcançar os objetivos traçados.

Doravante, o artigo focar-se-á na forma como foi materializada a automatização do processo de geração automática de armaduras e elaboração do Quadro de Pilares. Com efeito, pretende-se dar maior ênfase às potencialidades associadas à exploração da API do software e cujos fins / usos podem ser os mais diversos.

\section{Desafios}

O desenvolvimento de add-ins ou outras ferramentas informáticas para complemento, automatização e extensão das competências dos softwares BIM disponibilizados no mercado é um desafio atual e que julgamos será crescente nos tempos que se aproximam. A necessidade de otimizar a gestão da quantidade, cada vez maior, de informação gerada em cada processo, o aumento de produtividade exigido pela competitividade do mercado e a complexificação das geometrias associadas aos novos empreendimentos obrigam a um crescente domínio das competências associadas às ciências e tecnologias de programação.

A maioria dos softwares existentes no mercado promovem a possibilidade da criação dessas ferramentas através da utilização das API's. Contudo, estas API's requerem conhecimentos sólidos de programação e encontram-se escritas em linguagens de programação complexas como C\# ou C++.

A modelação geométrica dos elementos, num projeto de estruturas, revela-se como uma tarefa simples quando comparada com a modelação de armaduras ou a criação de plantas, cortes e detalhes com a informação necessária para a sua execução. A correta quantificação e elaboração de shop drawings com bar bending schedules depende da modelação de todas as armaduras, o que implica um acréscimo no tempo de modelação.

$\mathrm{Na}$ tarefa de filtrar os pilares no mesmo alinhamento vertical, com o objetivo de atribuir a mesma designação e comparar com outros pilares do modelo, não é possível automatizar com recurso às opções nativas do software. A opção Element Positioning, disponibilizada em Autodesk Revit ${ }^{\circledR}$ Extensions nas versões anteriores ao Autodesk Revit ${ }^{\circledR}$ 2019, permitia atribuir a designação dos elementos automaticamente, no entanto, distinguia os pilares não só por tipo como também por piso. A necessidade de ter os pilares do mesmo alinhamento agrupados e comparar esses conjuntos com base em determinadas propriedades é apenas possível de alcançar através da API do software. 
No fim pretendia-se que o aplicativo colocasse automaticamente o Quadro de Pilares referente ao modelo numa nova folha (Sheet) devidamente preenchida com vistas de pormenor dos diferentes pilares.

\section{Aplicativo}

O aplicativo foi desenvolvido com recurso ao Microsoft ${ }^{\circledR}$ Visual Studio 2015 permitindo, em ambiente Autodesk Revit ${ }^{\circledR}$, a execução e a validação do código. A utilização desta ferramenta prendeu-se com a necessidade de criar um aplicativo cuja interface fosse de fácil e intuitiva utilização. O aplicativo está dividido em três tarefas: (i) a geração de armaduras; (ii) a designação dos pilares e (iii) a elaboração do quadro de pilares. A geração de armaduras e a designação dos pilares podem ser realizadas sem qualquer ordem específica. $\mathrm{O}$ desenvolvimento do aplicativo implicou o acesso aos parâmetros (de instância ou tipo), à geometria dos pilares de betão armado e a métodos para criação de novos elementos, com a dificuldade inicial de compreender como a API do software disponibiliza essa informação. Essa informação serve de base para a geração de armaduras, assim como, para complementar a informação apresentada no quadro de pilares.

\subsection{Interface gráfica}

A interface gráfica utilizada é do tipo Windows Presentation Foundation (WPF) que exibe como principal vantagem a separação entre o design e o comportamento, promovendo a criação de um código melhor organizado. O design no WPF é criado geralmente no XAML (Extensible Application Markup Language) e o comportamento/código é implementado numa linguagem de programação [6], neste caso o C\#. Isto possibilita que a interface seja criada por um designer e o código por um programador, de maneira independente.

\subsection{Algoritmo}

A organização do código foi materializada à custa da criação de classes que agrupam propriedades e métodos para agilizar a interação com os diferentes propósitos do aplicativo. $\mathrm{O}$ aplicativo é dividido em três funções principais: geração de armaduras, designação dos pilares e criação de quadro de pilares com vistas de pormenor.

$\mathrm{Na}$ geração de armaduras existem diversas classes com o objetivo de recolher informações relativas à geometria do elemento, proceder à criação das armaduras horizontais e verticais e auxiliar na definição de métodos de cálculo. Previamente à criação de armaduras, o aplicativo realiza uma análise para que, no caso de o pilar ser intersetado por paredes e/ou lajes, seja executado automaticamente o comando Switch Join Order de modo a garantir que a sua geometria não seja alterada.

O aplicativo permite gerar armaduras para os pilares com a mesma geometria simultaneamente ou separadamente. O diâmetro e quantidade das armaduras verticais deve ser especificado pelo utilizador, assim como, o diâmetro e espaçamento do estribo exterior do pilar. Dependendo da quantidade de varões verticais, são gerados automaticamente estribos adicionais interiores. A geometria dos estribos interiores e a duplicação destes na zona de base e topo são criados com base em regras definidas no código do aplicativo.

$\mathrm{Na}$ tarefa de designação dos pilares houve a necessidade de filtrar os pilares e definir as propriedades a extrair. Esta tarefa pode ser realizada antes da geração de armaduras sendo que, 
se existirem objetos sem armadura, o aplicativo emite um alerta, prosseguindo se ter esse parâmetro em conta na diferenciação dos pilares. A classe ColumnsUtils foi criada especificamente para agrupar todas as operações relativas à designação dos pilares.

Inicialmente o algoritmo filtra os níveis presentes no modelo, ordenando-os por elevação de forma ascendente (ex. Nível -1, Nível 0, etc.). De seguida, para cada nível é criada uma listagem com os pilares em betão que estejam associados ao mesmo. Posteriormente, para cada pilar dessa listagem é criada uma "caixa delimitadora" (BoundingBox) que permite selecionar os pilares que se encontrem acima. A informação necessa'ria para a diferenciação de cada pilar, que se encontre dentro da "caixa delimitadora", é registada na classe ColumnsSet sendo cada ColumnsSet associado a um conjunto.

A classe ColumnsSet possui três listas que armazenam informação relativa aos pilares. A ListElement e a ListElementID contêm dados que são utilizados para acelerar as diferentes iterações no código. A ListElementInfo reúne informação como, o nível a que o pilar está associado, a sua geometria, a distância ao eixo e quantidade e diâmetro das armaduras. Esta informação permite comparar os diferentes conjuntos, com base na ListElementInfo, para encontrar os que são idênticos, atribuindo assim a mesma designação (Figura 1).
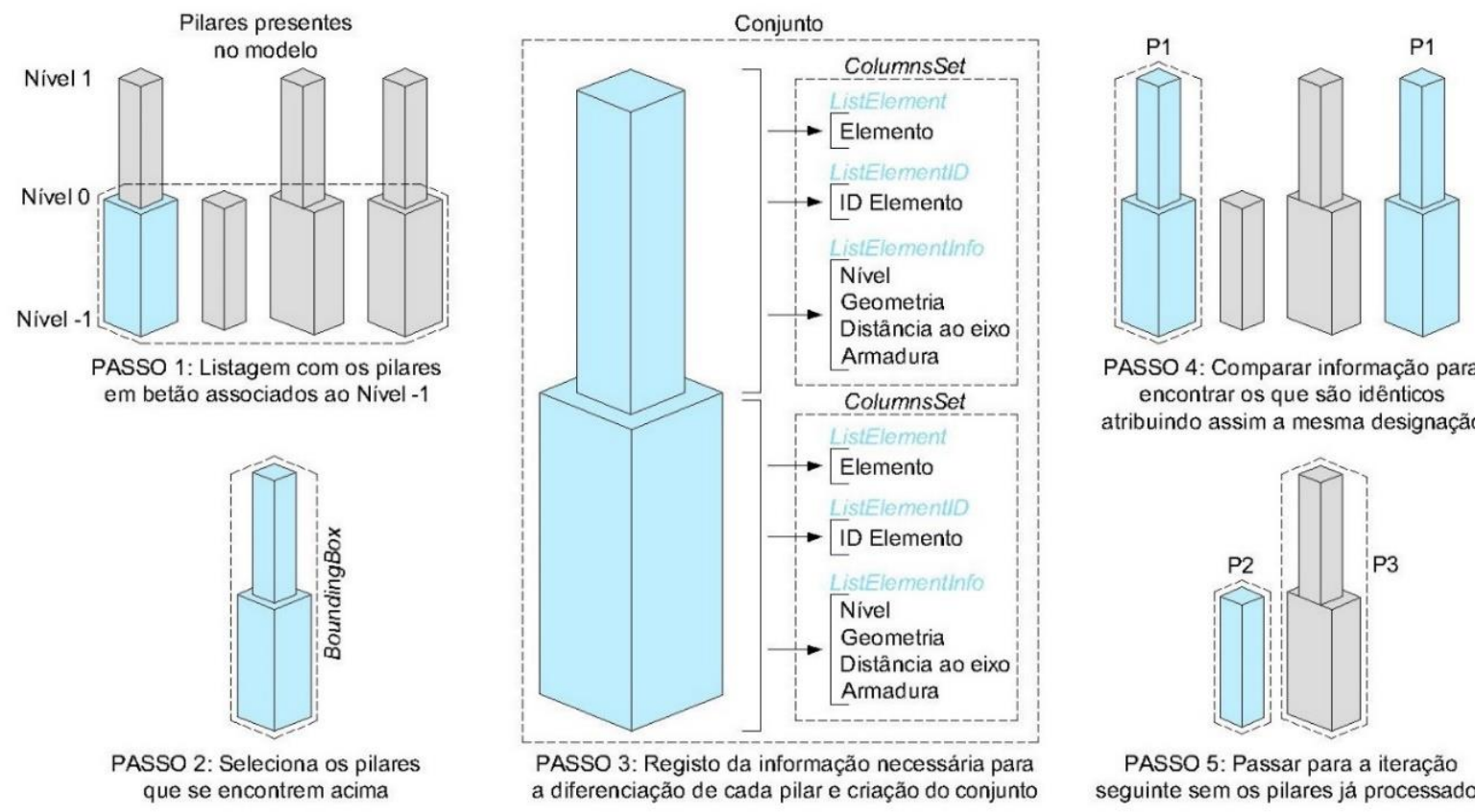

PASSO 4: Comparar informação para encontrar os que são idênticos atribuindo assim a mesma designação

Figura 1: Estrutura do processo.

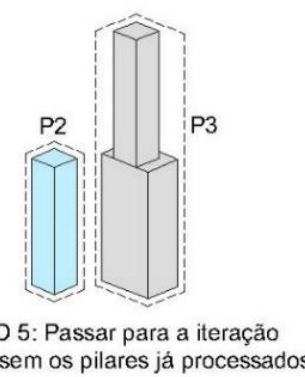

Em paralelo com a recolha de informação, foram criados métodos para determinar, caso a designação tenha sido introduzida / modificada manualmente, a existência de objetos sem armaduras e/ou sem identificação, conjuntos idênticos com identificação diferente ou se a numeração não é sequencial. Não obstante, o aplicativo reúne a informação relativa a cada situação e emite um alerta ao utilizador (Figura 2). Este processo funciona como um mecanismo de controlo do modelo. No entanto, se existirem pilares sem designação e/ou com designação incorreta, o aplicativo não avança se a situação identificada não for corrigida. 

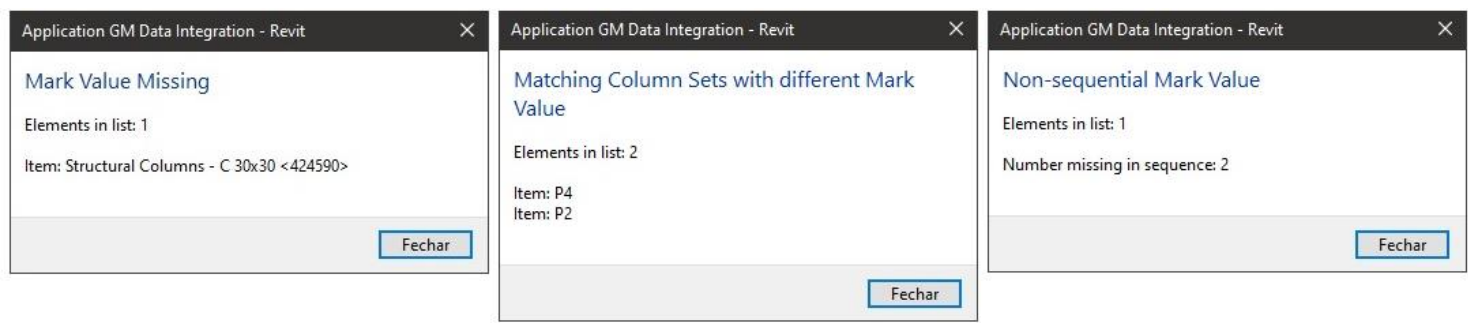

Figura 2: Mecanismo de controlo dos objetos.

Após validar a correta designação dos pilares o aplicativo cria, para cada pilar do conjunto, vistas de pormenor. As vistas incluem dimensões da geometria do pilar e a distância ao eixo, assim como informação relativa à armadura vertical e horizontal.

A esquadria do Quadro de Pilares é gerada recorrendo inteiramente à API do software, criando uma nova listagem (Schedules/ Quantities), opção nativa do Autodesk Revit ${ }^{\circledR}$, editando o cabeçalho de modo a poder inserir o número de linhas correspondente ao número de níveis presentes no modelo e colunas correspondentes ao número de designações de pilares diferentes. O Quadro de Pilares é inserido numa nova folha (Sheet), assim como as vistas de pormenor dos pilares.

\subsection{Utilização}

O aplicativo inicia apresentando uma listagem na qual é possível escolher de que forma os elementos são apresentados (Figura 3). O utilizador pode optar pela opção By Type que apresenta os diferentes tipos de pilares existentes no modelo. Na eventualidade de o utilizador pretender definir armadura para um pilar ou conjunto específico, pode selecionar a opção Selected ou By Set respetivamente. A opção Show elements with Reinforcement permite visualizar apenas os elementos que não possuem armaduras.

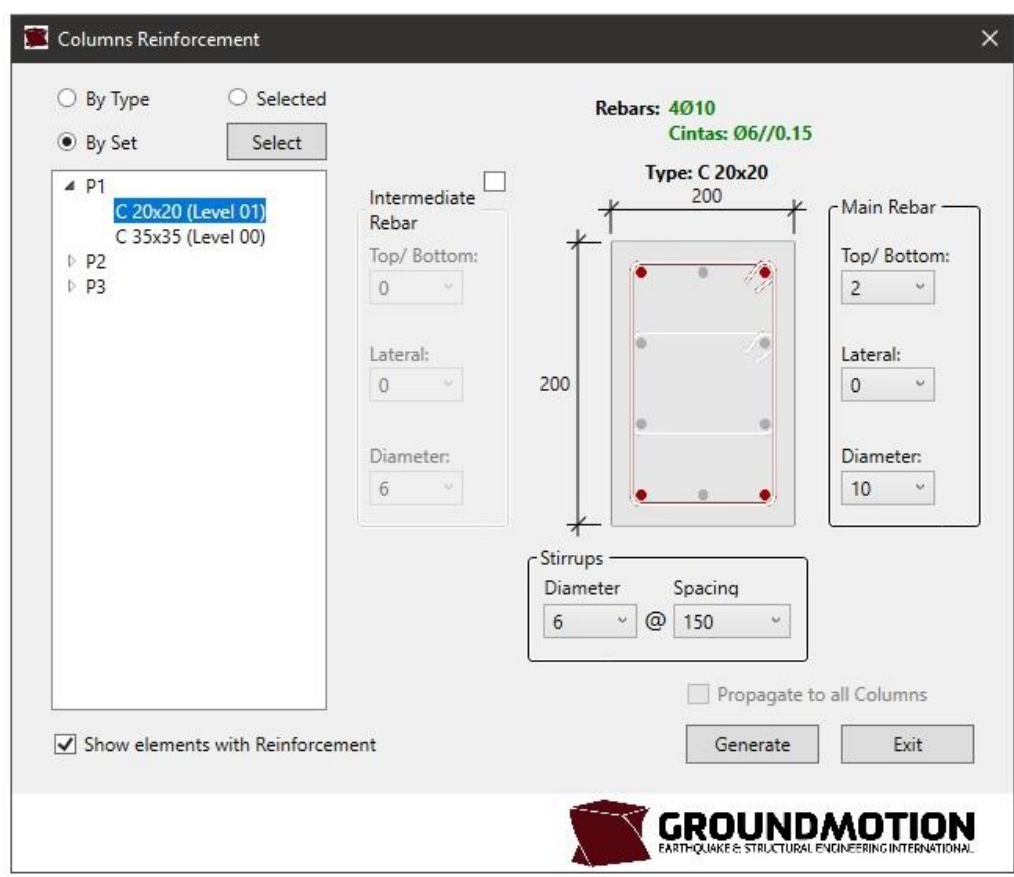

Figura 3: Interface do Aplicativo. 
Após a seleção do tipo de pilar, o utilizador deve especificar a armadura vertical e horizontal. $\mathrm{Na}$ armadura vertical é possível definir diâmetros diferentes. A interface contém campos de preenchimento para a armadura com o diâmetro maior à direita da seção do pilar (Main Rebar), onde se define o diâmetro e o número de varões para cada face do pilar. Caso exista, pode-se especificar, de igual forma, armadura com diâmetro menor do lado esquerdo (Intermediate Rebar). O diâmetro e espaçamento do estribo exterior deve ser especificado no campo Stirrups situado abaixo da seção do pilar. Os estribos interiores são gerados automaticamente com o mesmo diâmetro do exterior.

A interface disponibiliza a opção Propagate to all Columns que permite propagar a armadura definida para os pilares com a mesma geometria, independentemente da sua designação ou conjunto, sendo apenas possível para a opção By Type. Após a definição da armadura de cada tipo de pilar devemos selecionar a opção Generate de modo a gerar armadura especificada para todos os pilares/ conjuntos idênticos. Posteriormente a informação relativa à armadura será inserida em parâmetros de instância do pilar, de modo a agilizar a sua alteração e/ou replicar para outros elementos.

Uma outra interface foi desenvolvida para proceder à designação dos pilares. Esta interface disponibiliza ao utilizador a possibilidade de especificar o prefixo, o número inicial, o sufixo e o sentido XY no qual pretende iniciar a numeração.

A elaboração do quadro de pilares aparece como passo final do processo. O utilizador deve apenas especificar que níveis pretende incluir no quadro. As vistas de pormenor são geradas automaticamente e inseridas numa nova folha (Sheet), juntamente com o quadro, tendo como base a correspondência entre o nível e a designação do pilar. Na eventualidade de, para o nível especificado, não exista correspondência o aplicativo coloca uma linha no espaço vazio (Figura 4).

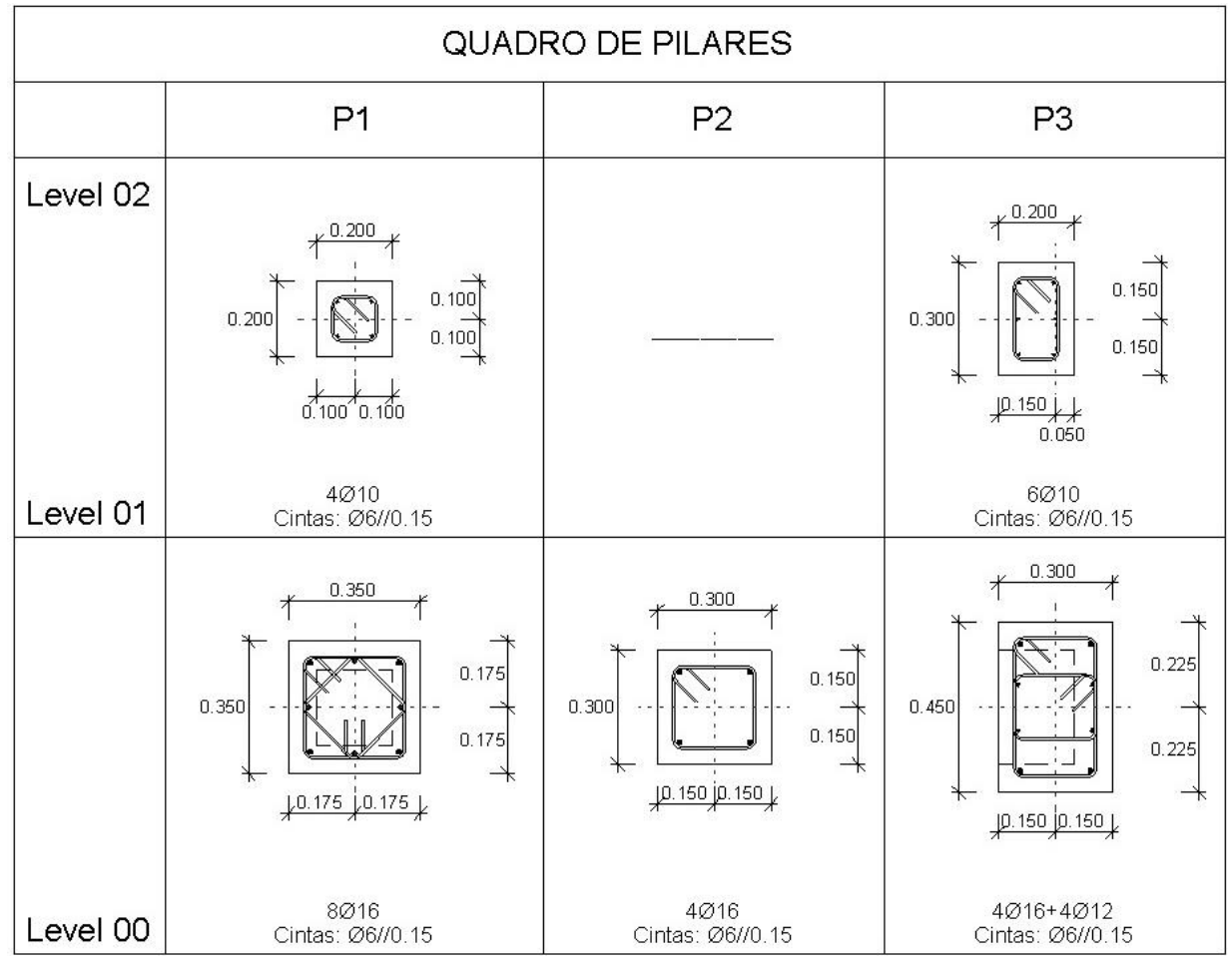

Figura 4: Quadro de pilares gerado automaticamente. 


\section{Limitações Atuais}

O aplicativo foi, nesta primeira fase, desenvolvido para agilizar a geração de armaduras, sendo que algumas das regras criadas para evitar alguns passos ao longo do processo possam resultar apenas para a maioria dos casos.

A pormenorização de armaduras, de acordo com templates empresariais padronizados, implica que a representação gráfica, como as anotações, tabelas ou propriedades incluídas nos modelos de vista (View Templates) sejam definidas manualmente. No entanto, estas definições podem ficar guardadas no template do software para utilização posterior em diferentes processos e modelos.

A determinação da informação necessária está indexada à designação dos elementos do modelo digital. Logo, o aplicativo recolhe informação apenas dos elementos devidamente designados. O aplicativo requere um nível mínimo de detalhe e de informação a ler nos objetos, i.e., é necessário que todos os pilares tenham designação e armaduras para a correta elaboração do Quadro de Pilares.

\section{Desenvolvimentos Futuros}

A lógica metodológica em que o aplicativo se baseia permite uma integração com diferentes softwares. Nessa perspetiva, poderão as competências do aplicativo ser alargadas para que as propriedades para a geração de armaduras possam ser especificadas em ambiente Microsoft ${ }^{\circledR}$ Excel ou Autodesk Robot ${ }^{\circledR}$ importando essa informação para o software Autodesk Revit ${ }^{\circledR}$.

O conhecimento adquirido da API do software na geração automatizada de armaduras servirá, no futuro, para extrapolar o procedimento para outros elementos de betão armado, possibilitando assim, um maior rigor na quantificação das armaduras e a criação automática de Shop Drawings com Bar Bending Schedules.

\section{Referências}

[1] Salman Azhar, "Building Information Modeling (BIM): Trends, Benefits, Risks, and Challenges for the AEC Industry" in Leadership and Management in Engineering (2011), Vol. 11, Issue 3, pp. 241-252.

[2] Marco Mellacqua, Elisa Bacchi e Davide Decarolis, "To Rebar or Not to Rebar: That Is the BIM Question", em Autodesk University, Las Vegas, 2017.

[3] BSI, "Scheduling, dimensioning, bending and cutting of steel reinforcement for concrete. Specification" (2005).

[4] Autodesk Knowledge Network - Development Requirements. URL: https://knowledge.autodesk.com/searchresult/caas/CloudHelp/cloudhelp/2018/ENU/Revit-API/files/GUID-FEF0ED40-86584C69-934D-7F83FB5D5B63-htm.html (consultado em novembro de 2017)

[5] P. Carvalho, "Avaliação do estado de implementação da tecnologia BIM no setor da construção em Portugal", Tese de Mestrado, Porto, FEUP, 2016.

[6] Visual Studio Documentation - Introdução ao WPF (Windows Presentation Foundation), 2016, URL: https://docs.microsoft.com/pt-br/visualstudio/designers/introduction-to-wpf (consultado em novembro 2017) 